\title{
Review of fiber optics technology for military applications
}

\section{Paul Ruffin}

Paul B. Ruffin, "Review of fiber optics technology for military applications," Proc. SPIE 10299, Novel Materials and Crystal Growth Techniques for Nonlinear Optical Devices: A Critical Review, 1029902 (23 June 2000); doi: 10.1117/12.419796

EDent: Symposium on High-Power Lasers and Applications, 2000, San Jose, SPIE. CA, United States 


\title{
A Review of Fiber Optics Technology for Military Applications
}

\author{
Paul B. Ruffin \\ US Army Aviation and Missile Command, Redstone Arsenal, AL 35898
}

\begin{abstract}
For the past two decades, fiber optics technology has been under development in the Army, Navy, and Air Force for applications, which range from fiber optic payout dispensers for air, land, and marine vehicles to fiber optic sensors for vehicle-navigation and environmental sensing and monitoring. Teleoperated weapon systems have been designed to provide the soldier with operator standoff capability, non-line-of-sight targeting, precision kill capability, with unmatched bandwidth. The Government/industry team conducted tremendous research efforts in developing a reliable fiber optic dispenser design, which is part of the data transmission link between a launch platform and a weapon. This paper reviews the advanced fiber optic technology, which includes automated fiber winding, and pack mechanics and payout dynamics models for producing stable fiber optic payout dispensers and thermally symmetric fiber optic gyroscope coils for operations in military environments. Also, non-destructive fiber optic techniques for measuring distributed strain and temperature in wound fiber packs will be discussed. The findings of the novel technique adopted for fiber pack quality verification are also discussed. Finally, the results of research efforts that are underway to develop low cost, high performance, miniature fiber optic gyroscopes for use in tactical weapon systems are presented.
\end{abstract}

Keywords: Fiber Optic Dispenser, High Speed Payout, Automated Fiber Winding, Fiber Optic Gyroscope, Dispenser Aging and Reliability, Fiber Pack Mechanics

\section{INTRODUCTION AND BACKGROUND}

The Corning Glass Works ${ }^{1}$ publication on the fabrication of single mode glass fiber with loss rates of $20 \mathrm{~dB} / \mathrm{km}$ in 1970 marks the birth of optical fiber for practical applications. Military applications for fiber optics have been primarily in the areas of fiber optic data links for guided vehicles and the fiber optic gyroscope technology. The concept of fiber guided vehicles, which was conceived in the U. S. Army Missile Command (MICOM) under the direction of Dr. William McCorkle, dates back as far as 1971. The notion to accurately hit a tank on the other side of a hill became a reality a decade later. ${ }^{2}$ MICOM initiated a program with Optelecom, Inc. in 1973 to demonstrate critical components for a fiber-optic data link that would allow non-line-of-sight communication to a Remotely Piloted Vehicle (RPV). ${ }^{3}$ In 1976, a 1-km step-index plastic clad silica fiber was successfully deployed from an RPV using a unidirectional mode of video transmission. In 1977, the feasibility of the fiber optic payout concept in military operational environments Was demenstrated at MLCOMA in

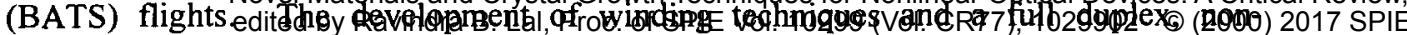
CCC code: $0277-786 X / 17 / \$ 18 \cdot$ doi: $10.1117 / 12.419796$

1

Proc. of SPIE Vol. 10299 1029902-1 


\section{2 / Critical Reviews Vol. CR77}

jammable data link to permit high-speed payout at longer ranges was conducted by the U. S. Army Communications and Electronics Command (CECOM) with Hughes Aircraft Company and ITT as contractors. The early fiber optic dispensers were tested at Holliman Air Force Base at speeds up to $500 \mathrm{ft} / \mathrm{s}$. Concurrent with the CECOM programs MICOM conducted a flight demonstration program (FOG-D) where duplex fiber-optic data links, which transmitted video camera images down to the ground station and control commands up the link to steer the missile, were implemented. Greater than $3-\mathrm{km}$ lengths of fiber were payed out at missile speeds.

In 1983, MICOM initiated the Fiber Optic Guided Missile (FOG-M) program, which was a proof-ofprincipal (POP) concept, to engage armored combat vehicles. The system consisted of a gunner's station and a pack of missiles mounted on a High Mobility Multipurpose Wheeled Vehicle (See Fig. 1). The gunner views the target and terrain via a seeker that is linked to the gunner's video monitor by a fiber optic cable. At least 14 missile flight tests were conducted where precision impact was made on moving as well as stationary targets using $10-\mathrm{km}$ multimode fibers links. The initial operational evaluation (IOE) of the FOG-M system and extended user

SINGLE FIBER PAYOUT

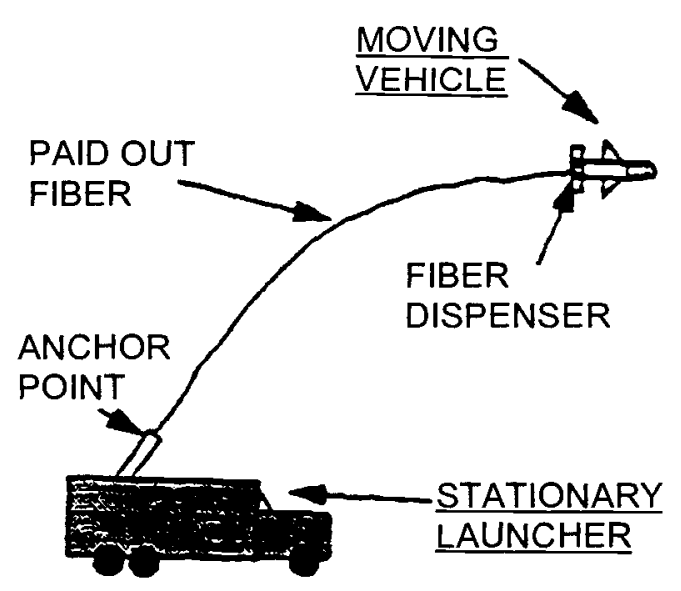

Figure 1: FOG-M Proof of Principle evaluation (EUE) tests were conducted from November 1988 to October 1990. The developments under the FOG-M program led to the non-line-of-sight (NLOS) program in 1987, where single mode fibers were used in data links exceeding $10-\mathrm{km}$ to provide defense against airborne vehicles. A full-scale development (FSD) contract was awarded to the Boeing/Hughes team in December 1988. Although the contract was terminated in January 1991, MICOM continued development for a NLOS system under the Non-Lineof-Sight Combined Arms (NLOS-CA) program. NLOS-CA was a precursor to the engineering and manufacturing development (EMD) program, which did not receive approval. In 1996, the Enhanced FOG-M (EFOG-M) program was initiated to engage armored combat vehicles, other high value ground targets, and helicoptors at ranges up to 1.5 times the original length of FOG-M. The Long-Range Fiber Optic Guidance (LONGFOG) program was initiated in the early 90's at MICOM to provide an extended range, multiple targets strike capability via a fiber optics data link. The Unmanned Ground Vehicles/Systems Joint Project Office (UGV/SJPO) demonstrated the first Army fiber-guided robot in 1997. In the mid-90's Dr. W. McCorkle at MICOM, currently the Aviation and Missile Command (AMCOM), devised a concept for the use of teleoperated missiles as a reconnaissance asset on the digital battlefield. ${ }^{4}$

The Navy has also been actively involved in the development of fiber optic guided weapons technology. The Naval Ocean Systems Center (NOSC) conducted the Sea Ray Project to develop single-mode, small diameter fiber, volumetrically efficient spooling techniques, and modeling to demonstrate long payouts from surface launched air 
vehicles. $^{5,6} \quad$ The Michelson Laboratory at the Naval Weapons Center (NWC) in China Lake, CA conducted the Sky Ray program, which demonstrated air-to-ground missile payout. The objective of the program was to demonstrate and document the performance characteristics of the fiber optic data link to assist industry in its development of competitive designs to meet the needs of the Navy. Dual fiber optic dispensers were simultaneously payed out from a weapon launched from an aircraft and from the launch aircraft as shown in Fig. 2. The dual payout technology was advanced under the Navy's Fiber Optic Guided Skipper (FOG-S) program, which adopts the Army's FOG-M technology to the Skipper weapons. ${ }^{7}$ FOG-S was concerned with system engineering integration of the seeker, fiber optic data link, propulsion, and control mechanizations from FOG-M to the Skipper weapon and defining system's interfaces. NOSC conducted a study to determine the achievable capabilities of fiber optic data links for Navy fiber guided systems. ${ }^{8}$

Marine applications for fiber-optic tethers include sonobuoys, torpedos, buoys and unmanned underwater vehicles (UUV's). The Naval Underwater Systems Center (NUSC) performed a study to develop technology required to deploy fiber optic dispensers underwater. ${ }^{9}$ These fiber optic dispensers are payed out at much lower speeds than air payouts but in a harsh environment. The inside payout approach as well as a pretwist fiber pack design is necessary for underwater payout.

The Air Force involvement in fiber-optic payout systems included the Guided Bomb Unit (GBU) $-15^{2}$ Eglin Air Force Base developed air- to-ground fiber optic payout using the GBU-15 missile.

This paper summarizes the fiber optic technology development in the military during the past two decades and presents more details on the programs mentioned above. A brief review of fiber payout fundamentals is presented in the next section. The results of studies performed to optimize the fiber pack design parameters via fiber pack mechanics and payout dynamics models/validation are presented in Sections 3 and 4 . The findings of a non-destructive measurement technique for fiber pack quality verification are also discussed. The progress of the fiber winding automation, which includes an automated adhesive application unit, is presented in Section 5. Fiber-optic dispenser aging and reliability issues are discussed in Section 6. Finally, the results of research efforts that are underway to develop low cost, high performance, miniature fiber optic gyroscopes for use in tactical weapon systems are presented in the last section. A novel winding technique, which eliminates fiber crossovers and provides for a thermally symmetric coil configuration, is also included. 


\section{FIBER OPTIC PAYOUT FUNDAMENTALS}

The basic fiber payout geometry is shown in Fig. 3, which illustrates a fiber optic dispenser mounted on a moving vehicle with the end of the fiber attached to a stationary launcher. The fiber is pulled out at a speed V. This conventional design approach is adopted for high-speed payout applications to avoid towing the fiber through the air, which causes the aerodynamic drag forces to increase beyond acceptable levels (exceeding the intrinsic strength of the fiber). Moving the fiber optic dispenser allows the fiber to quickly come to rest in the stationary surrounding air to maintain low tension. The tension $T_{1}$ in the fiber at the dispenser is given by ${ }^{10}$

$$
T_{1}=\rho V^{2}+F_{1}
$$

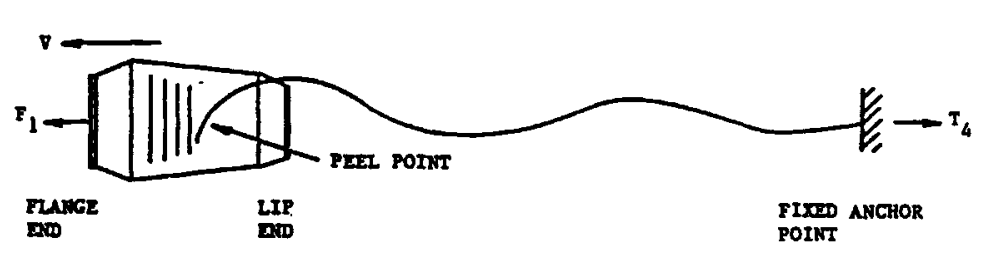

Figure 3: Payout Model where $\rho$ is the lineal fiber density, $V$ is the speed of the moving vehicle, and $F_{1}$ is the axial force caused by friction and

adhesive rupture at the peel point and the dragging of the fiber over the wound pack. Eq. 1 suggests that the aerodynamic drag-induced tension increases quadratically with the payout speed. The magnitude of $F_{1}$ is dictated by the payout dispenser design parameters (taper, adhesive, etc.). It depends upon the static (low speed) and dynamic (high-speed) effects.

The magnitudes of the first and second terms in Eq. I can be used as indicators as to whether a particular application is low, medium, or high speed. At very low speed $F_{1}$ is larger and the static forces are dominant. At very high speed $V^{2}$ is larger and the dynamic forces are dominant. The speed is medium when the first and second terms are on the same order. Low speed $(<25 \mathrm{ft} / \mathrm{s})$ applications include unmanned ground vehicles, etc. High speed ( $>250 \mathrm{ft} / \mathrm{s}$ ) applications include tactical missiles, etc. The maximum allowable speed is dictated by the fiber intrinsic strength. The fiber breaks when the tension (stress) exceeds the breaking strength.

The fiber comes off the fiber optic dispenser in the form of a rotating helix, where the diameter of the helix ( $\sim$ dispenser diameter) decays in an exponential manner a distance downstream the moving vehicle. The tension $T_{2}$ at the anchor point can be expressed as ${ }^{10}$

$$
\mathrm{T}_{2}=\left(1.5-\mathrm{C}_{1}\right) \mathrm{V}^{2}+\mathrm{C}_{2} \mathrm{~F}_{1}
$$

where $C_{1}$ and $C_{2}$ are constants, which depend on the payout geometry, bobbin diameter, fiber diameter, and fiber aerodynamic drag characteristics.

The fiber payout geometry for two moving vehicles is shown in Fig. 2. A single fiber optic dispenser is placed in each moving vehicle. This type payout approach is adopted for the scenario where a moving vehicle launches a second moving vehicle and move 
Novel Materials and Crystal Growth Techniques for Nonlinear Optical Devices / 5

away. Paying out the fiber from both ends reduces the aerodynamic drag force, which would be evident in the case of two moving vehicles. The two fiber optic dispensers are subjected to the tensions defined by Eq. 1 .

\section{FIBER OPTIC DISPENSER DEVELOPMENT ACTIVITIES}

\subsection{Payout Fiber}

Stringent physical and optical performance requirements are placed on the optical fiber that is wound under tension onto a cylindrical bobbin to produce an operationally-stable fiber pack to be deployed at high speeds $(>150 \mathrm{~m} / \mathrm{s})$ following a 10-year storage life under adverse military environments. The optical fiber, which is employed as a vital part of the data link, has a size on the order of a human hair as shown in Fig. 4. The payout fiber provides for a long-range, non-jammable data link. Imagery data from the guided weapon is transmitted down the link to the operator control station and control data is transmitted up the link for man-in-the-loop operations. This design enables remote control of the weapon from launch to impact. Sensors aboard the guided weapon permit real-time targeting decisions.

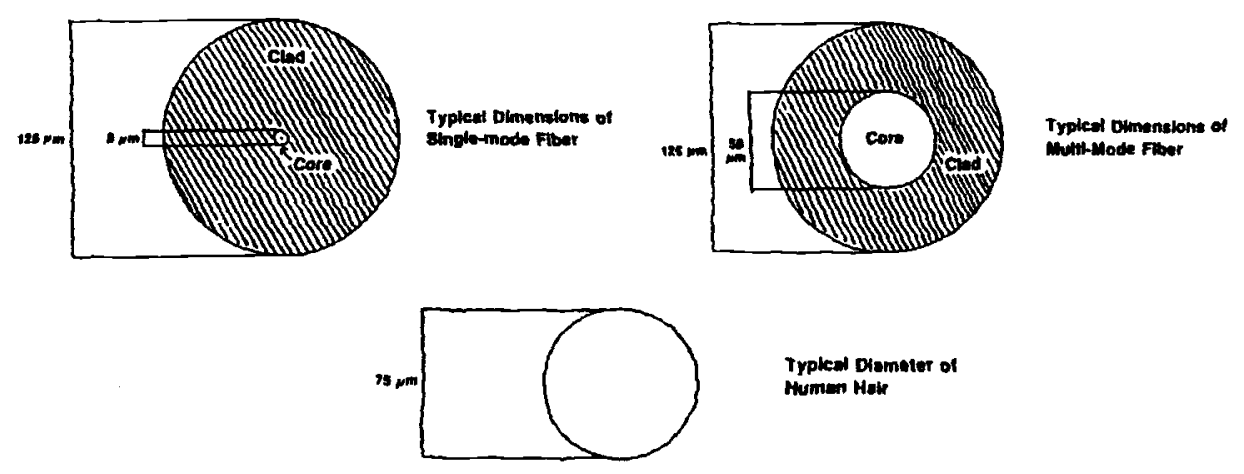

Figure 4: Fiber Size Comparisons

A special fiber with a duplex capability is required for the fiber optic data link to survive the harsh treatments during high-speed payout in military environments. Also, the payout fiber must exhibit resistance to bend-induced attenuation generated at the peel point (discussed in Section 4). The fibers most commonly used for payout applications are derived from telecommunications fibers.

The strength levels of payout fibers are established during the draw process by applying a specified "proof" tensile load to the fiber. The intrinsic or characteristic strength of the fiber is determined by conducting a series of tests where the fibers are pulled to failure at a specified rate. The intrinsic strength of high quality silica fiber is 1.4 times stronger than steel. However, the shear strength of the fiber is low.

The Government conducted programs to develop fibers of increased strength, greater resistance to bend induced attenuation, and stronger/thinner protective buffer layers. In the mid-70's low loss, step index, multimode fibers, operating in the $850-\mathrm{nm}$ regime, were used in the initial payout tests. In the late-70's, efforts were conducted to reduce the 


\section{6/ Critical Reviews Vol. CR77}

fiber core diameter from the standard 50 to $35 \mu \mathrm{m}$ for reduced optical attenuation at low temperature in wound fiber packs at increased lengths. The Integrated Fiber Optic Communications Link (IFOCL) program was initiated by CECOM with Hughes and ITT to develop the fiber optic data link. The IFOCL program led to the Enhanced Fiber Optic Communication Link (EFOCL) program, which was initiated to develop an advanced fiber optic data link. The initial fiber optic data link design, which was qualified for the FOG-M program was a $300 \mu \mathrm{m}$ O. D., $35 \mu \mathrm{m}$ core, and $200 \mathrm{kpsi}$ proof-tested fiber. In the mid-80's, efforts were conducted to develop, high strength, single mode fiber operating at longer wavelengths $(>1 \mu \mathrm{m})$ for extended range.

A standard measure of data link capacity is the length-bandwidth product. This lengthbandwidth parameter for standard off-the-shelf telecommunication fiber is $100 \mathrm{GHz}-\mathrm{km}$ at the $1550 \mathrm{~nm}$ wavelength. The fiber optic data link used in FOG-M required only a 125 MHz bandwidth capability.

Dispersion shifted (DS) fiber was considered to obtain low attenuation at both the 1.3 and $1.55 \mu \mathrm{m}$ wavelengths. ${ }^{11}$ Pre-twist degrades the attenuation performance in DS fibers at the $1.55 \mu \mathrm{m}$ wavelength. The DS fiber was replaced with a bend-insensitive fiber. Industry, AT\&T and Corning Glass Works, developed microbend insensitive, high- $\Delta$ single mode fibers. ${ }^{12,13} \Delta$ is the relative index difference between the core and cladding. The Naval Ocean Systems Center (NOSC) in Hawaii initiated a program to develop a dual-mode optical fiber for long-haul transmission. ${ }^{14}$ The matched-clad and depressedclad, pure-silica core designs were considered for applications in missile tethers and undersea surveillance cables.

Recently, a program was initiated at AMCOM, with contractors Spectran and Lucent Technologies, to develop a thin buffered $(200 \mu \mathrm{m})$ fiber for long range FOG-V systems. Reducing the fiber diameter from 250 to $200 \mu \mathrm{m}$ substantially reduces the number of layer in long-range fiber optic dispensers.

\subsection{Fiber Winding Methods}

The winding approaches considered for high-speed payout applications include: precision coil geometry, random winding, ortho-normal winding, basket-weave winding, flat coil geometry, hybrid coil geometry, and volumetric efficient winding. ${ }^{10}$ Since the winding dictates the payout characteristics, the winding must be accomplished such that dynamic pack instability is avoided. Winding defects lead to payout failures.

The precision coil geometry is used in most high-speed payout operations. The precision winding configuration involves winding the first layer of fiber onto a cylindrical bobbin in the form of a gentle helix which propagates with a pitch of one fiber diameter in the direction of the winding as shown in Fig. 5. Each fiber wrap is in contact with the preceding wrap. The direction of the pitch is changed during the transition from the first layer to the second layer when the direction of the winding is reversed as shown in Fig. 6. The fiber wraps of the second layer is nested in the grooves formed by the underlying wraps, except in the

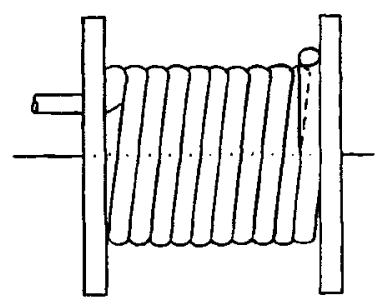

Figure 5: Winding Helix 
crossover regions. In cross section, the winding forms a hexagonal close pack pattern (See Fig 7). During the transition the fiber is stepped back over a specified number of underlying fibers, then settles into a new groove. There is a crossover event for each fiber wrap (See Fig. 6). Since the pitch of the winding is reversed, the fiber in the second layer is forced from one groove and obliquely crosses over two wraps of fiber in the first layer before settling into the proper groove for precision winding. ${ }^{10}$ This process continues for all layers wound above. The basic geometry for outside payout of the precision coil configuration is shown in Fig. 8.

The precision winding method, which has been proven successful in high-speed payout deployment applications, produces crossovers as discussed above. These unavoidable perturbations are prime candidates for fiber microbending. The precision winding method also subjects the fiber to combined tensile, radial (lateral), shear, and bending stresses, not recognized in straight fibers. These stresses conspire with the imperfections at the crossovers to cause more microbending which in turn causes coupling of fiber guided modes to radiation modes, resulting in energy leakage in multimode fiber.
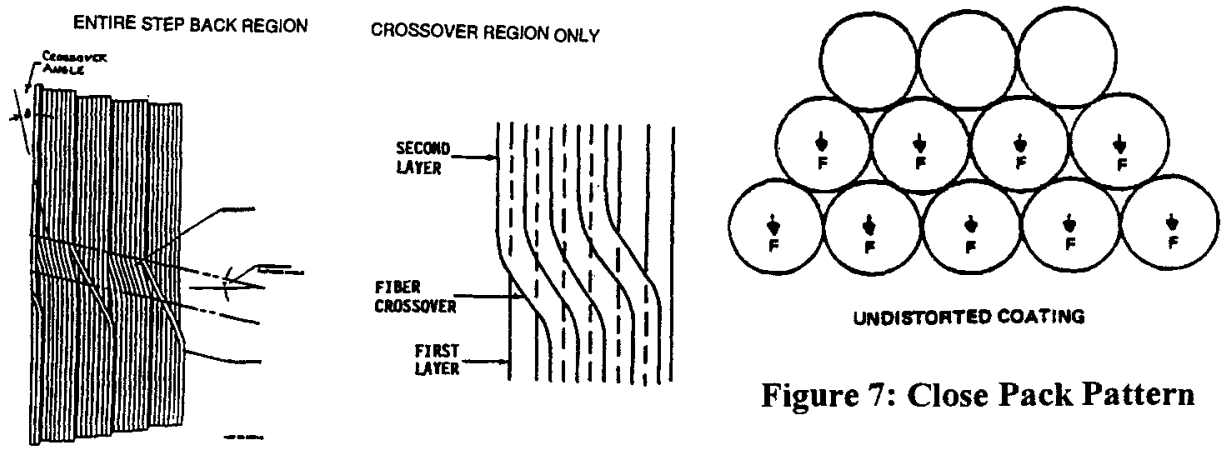

Figure 7: Close Pack Pattern

Figure 6: Stepbacks and Crossovers

Inside payout winding is accomplished via precision winding the fiber onto a bobbin, which is removed following the winding. The free fiber pack is mounted into an external container for payout operations. The fiber is paid out from the innermost layer of the wound pack as shown in Fig. 9. Advantages of inside payout include: no radially inward forces to disrupt the pack as in the case of outside payout, reduced frictional drag, and no bobbin which can cause thermal mismatch instabilities. Inside payout is more successful for underwater payout applications. The fiber packs for underwater applications are wound from flange to flange with no stepbacks. The inner diameter is less than 1.5 inches. A pre-twist is introduced to ensure smooth payout, which is free of fiber kinks.

Volumetrically efficient spooling (VES) involves winding the fiber flush to a single flange with step-backs only at the aft end. Reduced diameter $(135 \mu \mathrm{m})$ fiber is wound in a much smaller volume. Deep packs ( $>50$ layers) involve winding many layers of fiber in a compact configuration. 


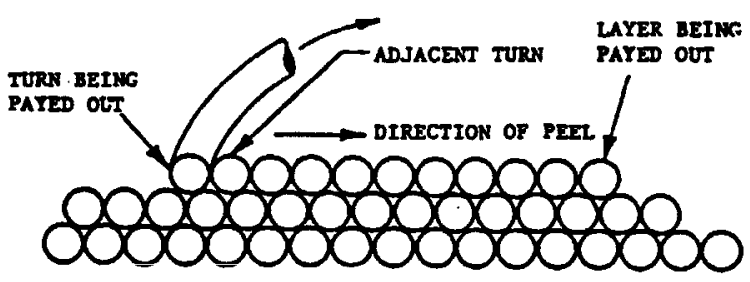

Figure 8: Outside Payout
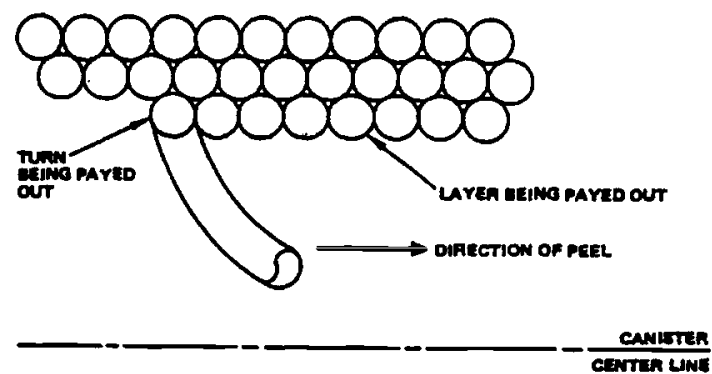

Figure 9: Inside Payout

\subsection{Fiber Cable Pack Mechanics Modeling}

Theoretical fiber pack mechanics models were developed by the Fiber Optic Guided Vehicle (FOG-V) team, consisting of Government and industry personnel, to confirm fiber pack stress conditions following winding. The long-term reliability of fiber optic payout dispensers is directly influenced by the residual tension distribution within the fiber pack and by the bending stress in the fiber at the crossovers. The early models provided an adequate mathematical description of the mechanical state of precisionwound fiber packs for the very unique instant that the winding is completed. The effects of bobbin stiffness, fiber buffer spring parameter, and winding tension parameters on the bobbin compression, base layer tension, and residual tension throughout the wound pack were determined for a freshly-wound fiber pack. The principal parameters of the model are:

- stiffness ratio of the bobbin to fiber

$$
\varepsilon=\mathrm{E}_{\mathrm{b}} \mathrm{A}_{\mathrm{b}} / \mathrm{EA}
$$

- transverse spring stiffness of the buffer coating $\quad \mathrm{K}=\mathrm{kR}^{2} / \mathrm{EA}$

- fiber winding tension

where $E_{b}$ is the Young's modulus of the bobbin, $A_{b}$ is the surface area of the bobbin, $E$ is the Young's modulus of the glass fiber, $A$ is the cross-sectional area of the fiber, $k$ is the squeeze test spring constant, and $\mathrm{R}$ is the bobbin radius. 
A set of determinate equilibrium equations for differential elements of the bobbin, base layer, and each fiber layer was solved via a tridiagonal computational process. Thus, the stress distribution in the fiber pack was determined. The early models predicted that stiffer bobbins, higher modulus fiber buffer coatings, and a wind tension profile were necessary to stabilize the pack during long-term storage and high-speed payout.

It is because of the role of the residual tension in resisting dynamic pack instability that a knowledge of pack mechanics is so important. A more complete set of factors influencing residual tension and pack stability are: 1) the winding tension profile, 2) the elastic and viscoelastic properties of the glass fiber, bobbin material, fiber buffer coating and adhesive materials, 3 ) the number of layers comprising the pack, 4) bobbin geometry, 5 ) the relative coefficients of thermal expansion (CTE) of glass fiber, bobbin, buffer coating and adhesive materials, 6) the temperature history and thermal state at the time of payout, and 7) degree of spacing between the fiber wraps.

The early aluminum bobbins contained a stainless steel base layer to provide grooves and dictate the spacing between the fiber wraps. These bobbins were inappropriate because of the CTE mismatch between the bobbin and the glass fiber. The thermal mismatch caused the inner layer fibers to loose tension, thus leading to much movement in the pack. The bobbin must be designed such that the CTE in the hoop (radial) direction match the CTE of the glass fiber and the CTE in the axial direction match the CTE of the buffer coating. It was also found that a grooved base layer as shown in Fig. 10 provided proper fiber spacing control

BOBBIN AND BASE LAYER DESIGN

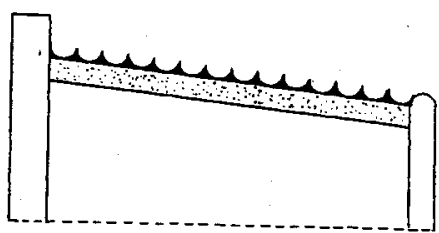

Figure 10: Grooved Baselaver and more stability. The grooves are formed via winding a precision, stainless steel wire onto a smooth, uncured epoxy surface, removing the wire and curing the epoxy.

Candidate materials to closely match glass include low expansion metal alloys, composites, sintered ceramics and injection molded plastics. The manufacturability of the bobbin with associated cost and physical properties such as compatibility with winding adhesives were considered in bobbin material selection. AMCOM-sponsored research produced several prototype bobbins of composite and metal alloy materials. These included graphite/epoxy, Kevlar-graphite/epoxy, and INVAR 36.

The early pack mechanics models suggested that the addition of more layers compress the lower layers, thus reducing their contribution to compression of the bobbin. Model validation techniques, described in the next section, were used to determine a proper tension profile to reduce or eliminate this effect.

The early fiber pack mechanics models described pack instability in a qualitative manner rather than in a quantitative manner. Analytical and experimental techniques were considered to determine the mechanical state of the precision-wound fiber packs following elevated temperature conditioning and storage. Results of laboratory experiments have shown that a heat conditioning and/or aging relieves a substantial portion of the residual stresses, especially in the outermost layers. ${ }^{15}$ 


\section{0 / Critical Reviews Vol. CR77}

The bending stresses at the crossover sites were not significantly reduced through a heat conditioning/aging process. A separate model was developed to investigate the stresses at the crossovers. The local inter-layer pressures and residual tension are input into the fiber crossover model. In the crossover region, the fiber is lifted from the groove in which it is nested between two rings of fiber in the layer beneath and crosses adjacent fibers at an angle, then becomes nested. The fiber tends to follow a helix on the fiber ring beneath. Since the fiber below is approximated as a torus, the configuration of the fiber in the crossover region behaves as a helix on a torus. A plane view of a typical crossover pattern in a fiber pack is illustrated in Fig. 6 . The bending stress $\sigma_{b}$ associated with the fiber geometry in the crossover region is expressed as ${ }^{16}$

$$
\sigma_{\mathrm{b}}=\left(\mathrm{E}_{\mathrm{f}} \mathrm{a}\right) / \rho
$$

where

$$
1 / \rho=\left[1+\left(r_{0} / d\right) \sin ^{2} \theta_{c}\right] /\left(r_{0}+d\right)
$$

is the fiber curvature, $E_{f}$ is the Young's modulus of the fiber, $a$ is the radius of the glass fiber, $r_{o}$ is the radius of the torus, $d$ is the diameter of the buffer coating, and $\theta_{c}$ is the crossover angle. The results of Eq. 5 are presented in Fig. 11 for a typical FOG-M fiber. The bending stress increases as the helix angle is increased. Abnormal crossovers can cause increased stress, thus reducing fiber pack reliability.

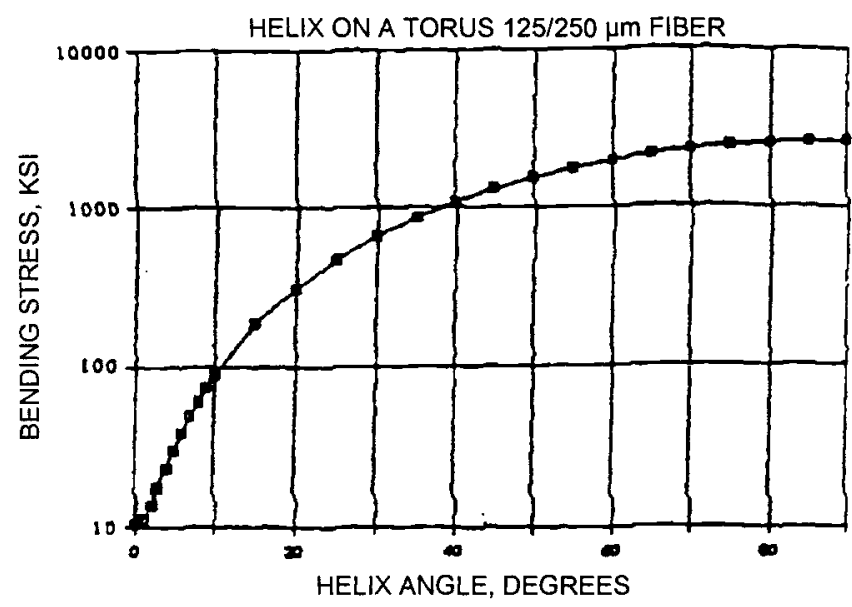

Figure 11: Helix Angle Bending Stress
The fiber crossovers are the prime contributors to excess loss in precision-wound optical fibers. A model was developed to predict the excess loss in a precision-wound paroboloc-index fiber. ${ }^{15}$ The model utilizes the coupled mode theory ${ }^{17}$ along with perturbation techniques at the fiber crossovers. The results of the model showed that a reduced core diameter substantially reduced microbending loss in the wound pack.

Furthermore it was determined that soaking precision-wound optical fibers at an elevated temperature for a proper period of time greatly reduces the initial excess signal loss. The elevated temperature soak, aimed at relaxing the internal shear stresses emanating from the winding process, brings the wound fiber pack to a stress equilibrium state in a short period of time (several hours). The model was extended to include the low temperature effects. ${ }^{18}$ Excess loss at low temperature is attributed to increased bending at the crossovers due to an increase in Young's modulus of the buffer coating at low temperature and increased radial pressure due to loss in tension in the inner layers. 
Novel Materials and Crystal Growth Techniques for Nonlinear Optical Devices / 11

\subsection{Adhesives Development}

An adhesive is frequently used to augment the stability of the pack against internal movement between the fiber wraps. In some cases (where the cable has high transverse stiffness) the adhesive is applied to the "stepback" regions only. In the case of soft cables, adhesive must be applied throughout the pack.

An adhesive is necessary to hold the fiber wraps in place as it is laid down during the winding process. The adhesive must stabilize the fiber wound pack over the full military range of environmental exposure and during high-speed payout. The adhesive must be compatible with the buffer, i. e. does not cause any long-term change in buffer characteristics due to chemical interactions between the buffer coating and adhesive (solvents). The adhesive must be applied uniformly to prevent irregular transient adhesive-rupture forces, which can cause fiber failure during payout. The CTE of the adhesive must also match the CTE of the buffer coating.

If there is sufficient residual fiber tension throughout the pack, the intrinsic stability is adequate without any adhesive (except to stabilize the layer end transitions). The adhesive augments the stability of the wound pack. Anything that tends to destroy the integrity of the adhesive within the pack also tends to reduce the augmented stability of the pack. As the fiber breaks away from the wound pack during high-speed payout there is a radially inward force on the fiber due to the curvature of the winding. The fiber has a tendency to dig into the pack and dislocates other fiber wraps, which can lead to fiber breakage. This effect is most likely to occur in the stepback region at the aft end of the wound pack. An adhesive is required to minimize the occurrence of this dynamic instability. The adhesive stabilizes the fiber wound pack against this failure mode (discussed in Section 6) as well as a resistance to relative motion between the fiber wraps during thermal cycles in storage.

The primary physical properties of winding adhesives include viscosity, modulus, peel strength, shear strength, cure rate, cure mechanism, dimensional uniformity, ductility, ease of application, effect on microbend attenuation, compatibility with fiber buffer coating, coefficient of friction, operational temperature range, environmental aging, effect on cable pack stresses and manufacturability of the pack in a cost efficient process. $^{19}$ A static peel force was used as a first indicator of a good adhesive formulation.

The use of adhesives results in increased fiber bending stresses at the peel point during payout. The adhesive strength must be bracketed between static and dynamic stability requirements. Inadequate adhesive strength results in dynamic instabilities (See Section 6). If the adhesive strength is excessive, peel point bending stress failures occur. This characteristic is illustrated in Fig. 12, where failure boundaries for these two failure mechanisms are plotted verses adhesive strength and payout speed. For successful payout, the operating point must be below the bending stress boundary and above the dynamic stability boundary. The distance from the operating point to either of the boundaries is a measure of the design margin against the particular failure mechanism. A sufficient design margin was incorporated into the fiber pack design for increased yield. 
It should be noted that the strength of most adhesives is strain-rate dependent. For the pack instability boundary, lowto-moderate strain rates are involved. For peel point bending, very high strain rates are present. As a result, for a given adhesive material and quantity, the operating point for adhesive strength is different for the two failure boundaries. The ideal adhesive would have a high strength at low strain rates, providing high pack stability,

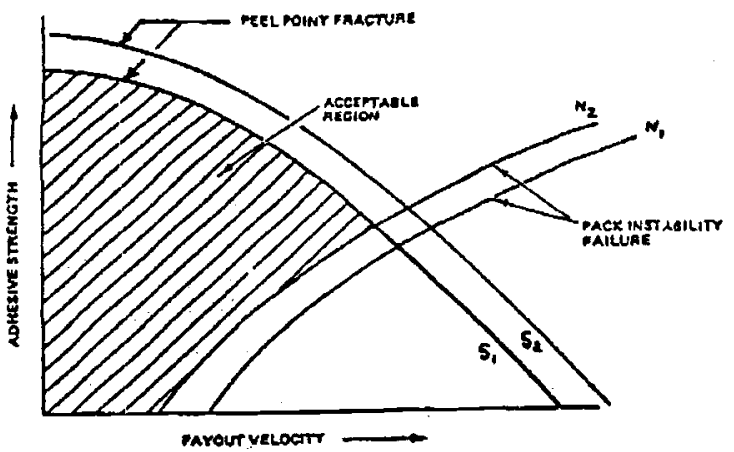

Figure 12: Adhesive Boundary Values but would have a low strength at high strain rates, resulting in low peel point bending stress. Unfortunately, for most adhesives, the high strain rate strength tends to be greater than that at low strain rates. This tendency tends to make the trade-off between these two failure modes even more difficult.

Through 1987, the types of adhesive investigated for fiber optic dispensers were neoprene, in the form of Polyfoam Rubber Cement and/or Lockbond 116 (Standard Brands). ${ }^{20}$ Although successful payouts were conducted under ambient conditions, these adhesives exhibited poor aging characteristics when subjected to environmental temperature limits. Advanced adhesives, which include V-glue, Dow Corning Q2-7406, and UV-curable adhesives, were investigated for the long-range FOG-V systems. AMCOM initiated efforts in 1996 to develop a solventless adhesive material for use in EFOGM. $^{21}$

\subsection{Model Validation}

Several instruments were investigated to measure strain in fiber optic dispensers. A Strain Sensing Optical Time Domain Reflectometer (SSOTDR) was developed by Georgia Tech to measure the total strain changes in a wound fiber pack. ${ }^{22}$ The SSOTDR was the first non-destructive measurement device, which was employed at AMCOM as a tool to validate fiber cable pack mechanics models via empirical determination of the residual energies (tensions) in wound fiber packs. The SSOTDR was used in concert with a rotary fiber coupler to obtain real-time fiber strain data during the precision winding process. The collective strain measurement yielded the sum of the fiber strains per layer and provided an indication of the equilibrium tensions generated by winding multiple layers of optical fiber. During thermal cycling, the viscoelastic response of the fiber allowed tensions within the pack to relax. The SSOTDR provided a direct method of capturing this relaxation phenomenon in wound fiber packs.

Since the SSOTDR did not provide fiber length-dependent strain capabilities, an advanced, non-destructive measurement capability was pursued to measure the distributed strain throughout the layers of the wound pack. A Brillouin Optical Time Domain Reflectometer (BOTDR), which is manufactured by Ando Corporation, was evaluated and found to be the leading candidate for measuring distributed strain in fiber optic dispensers. ${ }^{23}$ The BOTDR utilizes stimulated Brillouin scattering (SBS), which is a 
nonlinear interaction between light-waves and acoustic waves. Distributed strain plots were generated for fibers wound under various configurations (fiber optic dispensers and fiber optic gyroscopes). Comparisons were made between model predictions and measured data for a number of winding scenarios. ${ }^{24}$ Fiber layers, step-backs, winding anomalies, pack deformations and aging were discernable.

BOTDR measurements at cold temperature revealed that the outside layers of the fiber optic dispenser were in compression. ${ }^{25}$ Furthermore, it was determined that the Brillouin Coefficient of the fiber buffer coating was not linear for cold temperatures.

\section{HIGH SPEED PAYOUT ACTIVITIES}

\subsection{Payout Dynamics Modeling and Validation}

The FOG-V team adopted Padfield's ${ }^{26}$ model for the dynamics of a flexible string and developed computer models to predict the behavior of the optical fiber during high speed payout. Knowledge of the bend geometry of the peel point was necessary for designing the optimum payout fiber. The peel point is a sharp bend where the fiber being payed out leaves the wound pack. Additional stress is exerted on the fiber at the peel point during high-speed payout. This stress is maintained for less than a millisecond. The curvature and bend stress at the peel point is directly related to the fiber stiffness, adhesive bonding, and the friction caused by the fiber moving over the wound pack beneath.

The fiber shape at the peel point during high speed, steady-state payout was obtained analytically by applying Newton's second law to a fiber, which moves on the surface of a cylindrical bobbin, without friction, in the presence of an adhesive force. The equations of motion were solved analytically to obtain a closed form solution, which describes the shape of the fiber from the peel point to the point where it leaves the bobbin. The resulting equation for the case of a concentrated adhesive force is ${ }^{27,28}$

where

$$
\phi=\alpha-4 \arctan \left\{\tan (\alpha / 4) \mathrm{e}^{-\sigma \mathrm{A}}\right\}
$$

$$
A=\left(T_{t o}-m_{0} V^{2}\right) / E_{c} I_{c},
$$

$\phi$ is the angle formed by a unit vector tangent to the fiber and the bobbin axis, $\alpha$ is the value of $\phi$ at the takeoff point, $\sigma$ is the distance along fiber from peel point, $T_{\text {to }}$ is the fiber tension, $m_{0}$ is the lineal mass density of the fiber, $V$ is the payout velocity of the fiber, and $E_{c} I_{c}$ is the flexual rigidity of the fiber. The maximum bending stress can be expressed as

$$
\sigma_{\mathrm{f}}=\mathrm{Ed} / 2 \delta_{\mathrm{o}}
$$

where $\mathrm{E}$ is the Young's modulus of the composite fiber, $\mathrm{d}$ is the fiber diameter, and $\delta_{0}$ is the minimum radius of curvature.

AMCOM conducted a series of aircraft payout tests at the Naval Surface Warfare Center in China Lake to obtain data to validate the fiber payout dynamics model. A high-speed image capture system, described in the next section, was developed and implemented 
during the tests. Two-dimensional peel point images were obtained to provide a tool for understanding the dynamics of optical fiber payout. The photographic data were compared to the model for peel point shape for both hardway peel - the fiber is payed out from the forward end of the bobbin and easyway peel - the fiber is payed out from the aft end of the bobbin to the forward end of the bobbin as illustrated in Fig. 13.

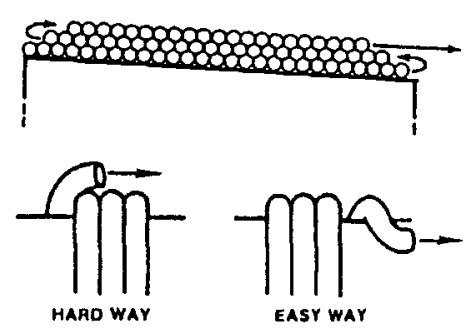

Figure 13: Peel Point

\subsection{High Speed Payout Test Platforms/Instrumentation}

Several high-speed payout machines were designed and constructed for the purpose of investigating payout dynamics. Instrumented laboratory and aircraft (Caribou, TA-7, C-130, F-16) were utilized to evaluate various fiber optic dispenser designs. Pinch-Wheels and P-Shoe Payout Machines were adopted for most of the laboratory payouts. The aerodynamic drag on the fiber in the laboratory represents the worse case scenario, since the fiber is pulled through the air for greater than $50 \mathrm{ft}$ to damp out the helix before entering the payout machine eyelet. The laboratory instruments include a Force and Torque Sensing Spool Fixture (FTSF), a Fiber Turns

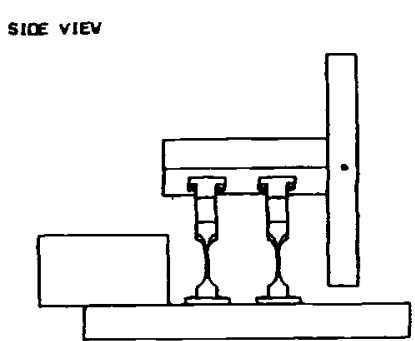

Figure 14: Force and Torque Sensing Fixture

Counter Device, a pulsed or continuous wave optical source coupled into one end of the fiber and a photodetector at the far end, a Bit Error Rate Tester, Strobe Photography, and High Speed Video.

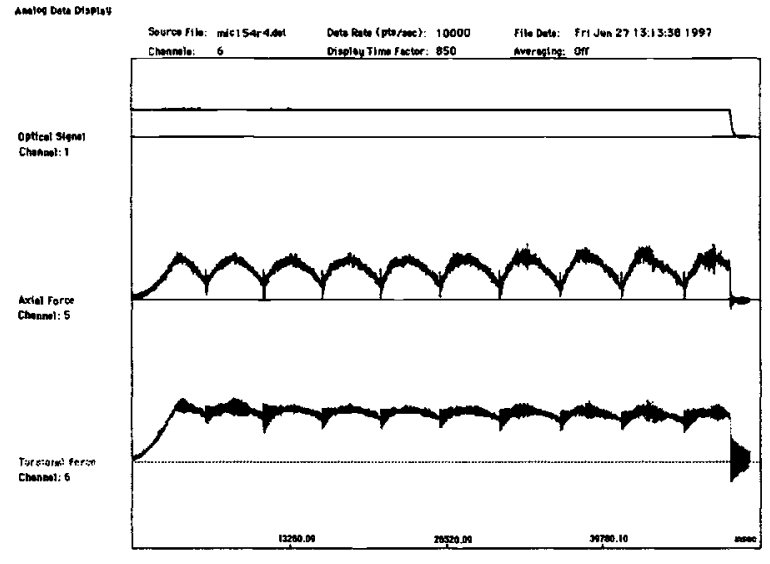

Figure 15: Axial and Torsional Forces
The Force and Torque Sensing Spool Fixture, shown in Fig. 14, was used to measure the bobbin strains. The axial force $F_{1}$ in Eq. 1 varies (in a smooth manner) as the peel point moves back and forth along the wound pack as shown in Fig. 15. The minimum point on the trace represent the axial force when the peel point is at the aft end of the tapered fiber pack where there is no contact with the pack. The maximum point on the trace represent the axial force when the peel point is at the forward end of the fiber pack where the fiber is being dragged over the entire length of the fiber pack. Adjusting the adhesive and bobbin taper can alter the measurement. 
The Fiber Turns Counter Device, shown in Fig. 16, was used to provide a direct measure of the fiber payout velocity. A photointerrupter scheme is adopted where an optical device senses the fiber wraps as they are removed from the bobbin. ${ }^{29}$ An infrared LED is mounted on the inner surface of the fiber optic dispenser and a photodetector is mounted on an extended arm alongside the fiber optic dispenser. The velocity is accomplished via counting the number

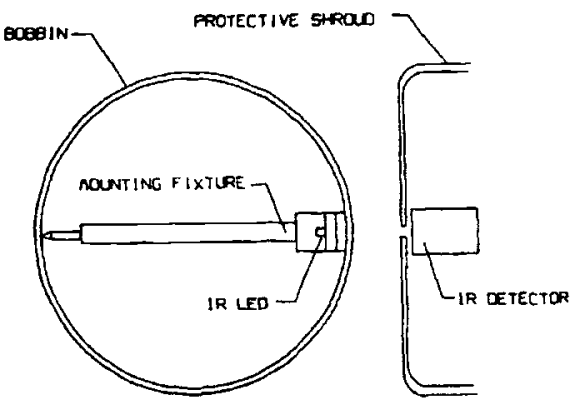

Figure 16: Turns Counter of times the optical path is broken by the payout fiber and multiplied by the length of each fiber loop.

A high-speed image capture system was developed and implemented on a jet aircraft platform to obtain two-dimensional peel point images and instabilities.

\section{AUTOMATED FIBER WINDING PROJECTS}

A great deal of effort was devoted to automating the fiber winding process. In 1983, MICOM initiated a technology base project to automate the winding process for fiber-optic payout dispensers. ${ }^{30,31,32}$ A breadboard (BB) winder was constructed to serve as a base for automation. The basic requirements for the winder are to construct mechanically stable fiber packs for high-speed payout. The BB winder was referred to as the Engineering Development Model (EDM). The winder is comprised of a platform 22 for supporting the bobbin 16 , a motor 24 for rotating the bobbin, a motor 26 for traversing the platform, a tension unit 17 , a supply spool 12 , an

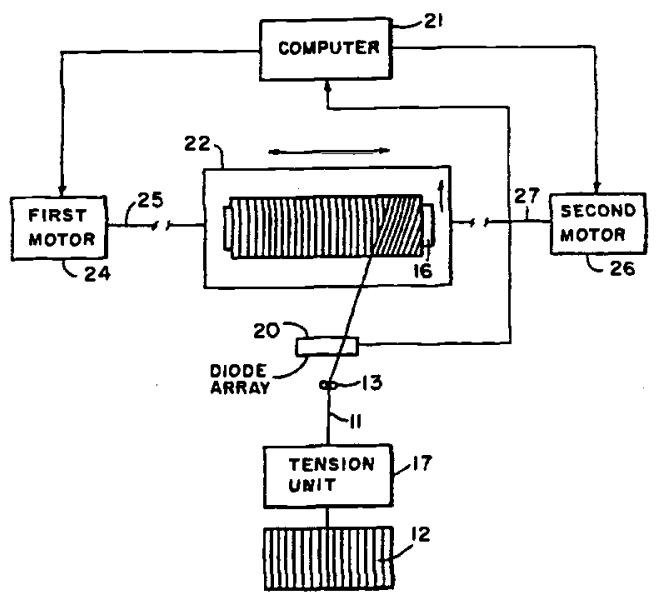

Figure 17: Fiber Winder eyelet 13 , a CCD array 20 , and a computer 21 as shown in Fig $17 .^{33}$

The second generation winder, known as the Production Prototype Model (PPM), incorporated machine vision systems capability to monitor fiber lay-down to permit inspection, detection, and correction of winding flaws. The machine vision system allowed the step back wraps at the bobbin transition regions to be accomplished automatically. Another feature of the PPM was the incorporation of a single mode rotary coupler on the fiber feed spool to provide optical signal monitoring capability. More than $7 \mathrm{~km}$ of fiber was wound on the PPM automatically. 
The third generation winder features an Active Lag Angle Device (ALAD), which allows on-line adhesive application and better control of the fiber stepback process. An automated adhesive application system was developed and transferred to industry. ${ }^{34}$

A significant milestone in the fiber winding automation process was the production of precision controlled fiber diameters. The initial fibers had a diameter variation specification of $+/-6 \%$. The most recent fiber has a diameter variation of less than $1 \%$. An Optical Fiber Inspection System was developed to inspect the incoming fibers for buffer flaws prior to winding to ensure uniformity.

The Chambered Adhesive Spray Application (CASA) was a key to full automation. The winding automation efforts resulted in a 10-fold reduction in winding time.

\section{FIBER OPTIC DISPENSER RELIABILITY}

Because of the lack of detailed understanding of peel point bending stresses, the corresponding degradation of fiber dispenser reliability is difficult to establish. Because of the high levels required for operational dispensers, however, it becomes important to identify and such degradation.

Eq. 1 suggests that the tendency for dynamic pack instability increases as payout speed is increased. Dynamic pack instability, which is triggered by payout induced forces in the fiber near the peel point, can cause fiber breakage during high-speed payout. There are two known dynamic pack instability failure modes associated with high speed payout dispensers: multiple turn dispense from the middle parts of a wound layer and the knifing of the fiber down into the pack creating snarls and tangles in the fiber. The first dynamic pack instability failure mode results in a disturbance and potential fiber breakage aft of the peel point along the surface of the wound pack. The second fiber failure mechanism is a catastrophic disturbance in the vicinity of the peel point.

\subsection{Fiber Dispenser Aging Mechanisms}

There are a number of aging mechanisms, which may potentially limit the storage and operational lifetime of the fiber optic dispenser. ${ }^{35}$ An accelerated aging test plan was developed by the FOG-V team. ${ }^{36}$ The aging mechanisms associated with fiber optic payout dispensers can be grouped into the following classifications:

1) Chemical Aging of Buffer and Adhesive

2) Mechanical

3) Static Fatigue Effects on Fiber Strength

The first category is subject to investigation through standard accelerated aging techniques based on the Arrhenus model. The chemical changes that may occur within a fiber optic dispenser include oxidation, cross-linking, hydrolysis, polymerization and depolymerization, outgassing, and chemical interaction between adhesive and buffer materials.

The second category includes normal mechanical degradation associated with shock and vibration. The visco-elastic effects within the fiber pack are included. The physical 
parameters of the adhesive and buffer materials, which influence the optical and mechanical performance of the fiber optic dispenser and are believed to be subject to aging-induced changes include: modulus, adhesion (buffer/glass and buffer/adhesive), elongation, friction, mechanical strength, and influences on fiber strength.

The third category relates to non-Arrenius processes, which may be accelerated, but the acceleration factors are subject to wide uncertainties. The fiber crossovers are prime sites for static fatigue in high humidity environments. It is well known that the intrinsic strength of optical fibers degrades when stored under a sufficient amount of stress in humid environments.

A number of "static" disturbances of the wound pack have been observed during simulated bobbin aging. Fiber pack "slumping" (See Fig. 18) is a well-known pack defect. Other static mechanisms observed include "earthquakes", birch-bark, and fissures as shown in Fig. 19. Most of these static mechanisms involve significant straining of the adhesive in the vicinity of the disturbance. An adhesive rupture is obvious in the case of an "earthquake". When the integrity of the adhesive is locally destroyed it creates "fissures" in which gaps have opened in the pack but subsequently closed. In the case of "birch-bark" it is likely that the adhesive integrity has been locally degraded.

EXAMPLES OF PACK DEFECTS SLUMPS

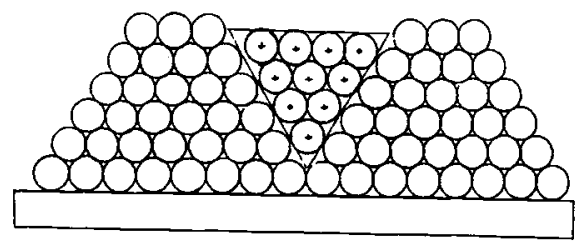

Figure 18: Pack Slumps
EXAMPLES OF PACK DEFECTS CRACKS

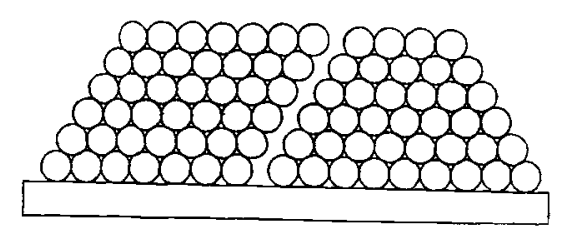

Figure 19: Pack Fissures

\subsection{Fiber Failure Mechanisms during Dispense}

The mechanisms, which are responsible for fiber failure during high-speed payout, are briefly discussed here. ${ }^{14}$ 1) The force required to rupture the adhesive results in an axial force coupled into the fiber wraps adjacent to the fiber being payed out. This force, which is in the direction of the aft end of the wound pack, tends to axially compress the fiber wraps in the layers beneath. 2) The fiber being payed out contacts the downstream fiber in the layer beneath as it is lifted out of its nesting position in the pack for easy-way peel and as it is lifted over the adjacent fiber wrap for hard-way peel at the peel point. 3) The fiber tension at the peel point propagates for a short distance ahead of the peel point, decaying roughly exponentially due to friction and distortion of the adhesive. This region of increased tension coupled with the circular geometry of the fiber wrap results in a tendency for the fiber being payed out to knife down into the lower layers, generating both axial and radial force components in the fiber pack. 
Fiber failure mechanism \# 1 above leads to multiple fiber-wrap dispenses, which occasionally occurs simultaneously from more than one layer. Snarls and tangles can occur. This mode is triggered by the axial force disturbance trying to force the fiber wraps in the lower layer toward the aft end of the wound pack. One or more of the fiber wraps being forced up out of the fiber pack can relieve the axially compressive force. These disturbances, which usually extend only a short distance around the surface of the wound pack, are called "pop-ups". These popped-up zones are subject to buffeting as the rotating helix of the fiber being payed out impacts them with every rotation. The disturbance can become knocked out of position and entangled with the rotating helix, resulting in multiple wrap payout, sometimes from more than one layer. The signature of this type failure mode is a region of local snarls and tangles, which is displaced down stream from the peel point.

Fiber failure mechanisms \# 3 above leads to a catastrophic disturbance in the vicinity of the peel point. There is a tendency for the fiber wrap being payed out to be pulled down into the pack beneath. The resulting forces from the fiber trying to climb back up can explosively tear the pack apart in a local region. The signature of this failure mode is a snarl or tangle in the immediate vicinity of the peel point.

The mechanisms, which trigger the dynamic pack instability, are present in every highspeed payout. The movement of the fiber wraps, which triggers instability failures, tends to be abrupt and relatively large. Residual tension in the layers beneath tends to resist movement in the pack. The resulting stability is called intrinsic pack stability. An adhesive binder also augments the stability of the pack against unwanted movement.

\section{FIBER OPTIC GYROSCOPE DEVELOPMENT}

The operation of the interferometric fiber optic gyroscope (IFOG) is based on a Sagnac interferometer in which a beam of light is split and sent in both directions through the sensing coil (See Fig. 20). After traveling the length of coiled fiber, the counterpropagating beams are recombined to form an interference pattern, which shifts when a rotation is sensed. Rotation is sensed because the two beams encounter different path lengths when the coil is rotated.

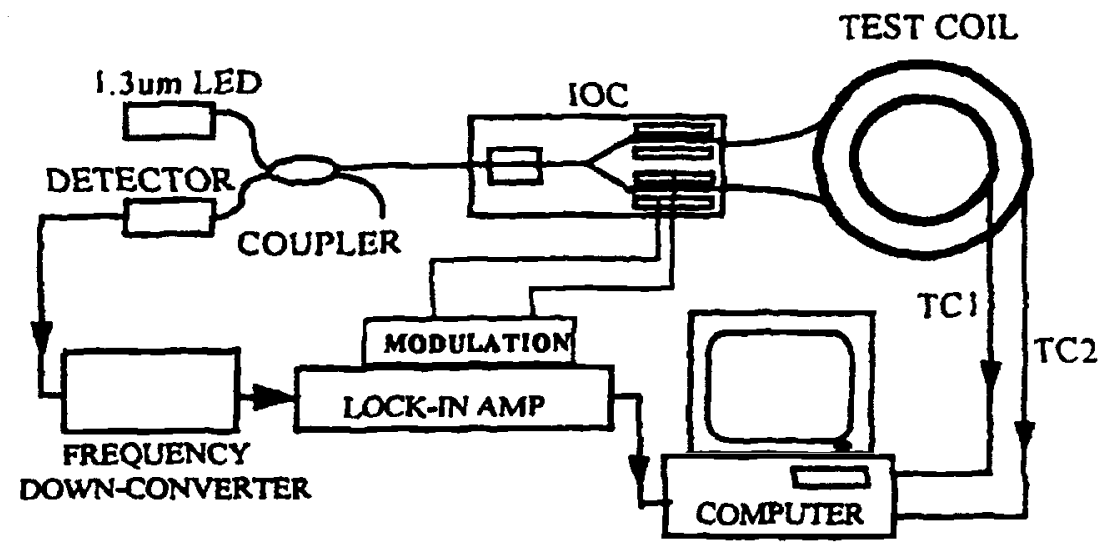

Figure 20: Fiber Optic Gyroscope 
Tactical weapon systems require small, low cost, reliable, inertial measurement units to meet the stringent environmental performance requirements emanating from a diverse range of applications. The IFOG, which has reached a level of practical use in navigation, guidance and control of aircraft, missiles, automobiles, etc., has been shown to meet most of these requirements. ${ }^{37}$

Tremendous progress has been made during the past decade in developing high performance light source modules operating in the near infrared region, environmentally robust packaging schemes for the sensor coils assembly, and automated, precision techniques for the interconnection of miniature optical components and FOG subassemblies. ${ }^{38,39,40}$ Advanced research and development programs, that are intended to reduce the cost of critical components/manufacturing processes related to FOG production via the development of enhanced manufacturing processes and techniques to enhance the US navigation industrial base, are currently underway. ${ }^{41}$ While these FOG component-level technology advances and cost effective processes for assembling the FOG systems meet most of the requirements for tactical weapon systems, the cost and technical issues associated with ultraminiature FOG designs are just recently being addressed by AMCOM.

The FOG optical components are inherently small, except perhaps the sensor coil. The small size limitations for the sensing coil and the optimization of an ultraminiature FOG for operation in military environments have typically been neglected in the literature. Further attention should be given to the performance and reliability aspects of ultraminiature coil designs and the packaging of miniature FOG components.

The performance of IFOG has been shown to be sensitive to time-varying thermal gradients present across the fiber optic-sensing coil. The non-reciprocity described by Shupe ${ }^{42}$ causes a false rotation signal when the counter-propagating beams encounter sections of fiber with time-varying thermal gradients and no longer travel identical paths through the coil, seen in the precision wind configuration shown in Fig 21. Frigo ${ }^{43}$ proposed a quadrupolar winding technique, in which the fiber is wound from the center point, distributing the thermal gradients symmetrically across the length of fiber as shown in Fig. 22. This technique has been widely adopted for IFOGs. In the mid-1980's coil geometry and packaging techniques for minimizing thermal gradients were presented. ${ }^{44}$ Temperature rate-induced IFOG bias was measured at Honeywell over a limited temperature range. ${ }^{45}$ A preliminary comparison between quadrupolar and straight winds has been made by Smiths Industries and AMCOM. ${ }^{46}$

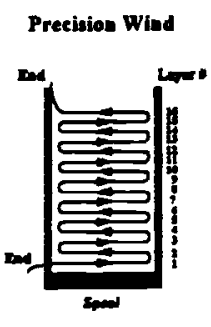

Figure 21: Precision Wind

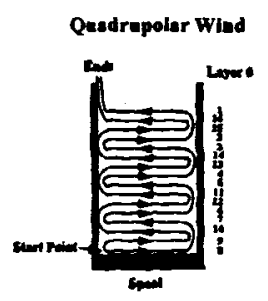

Figure 22: Quadrupolar Wind

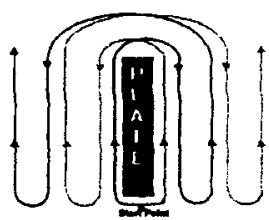

Figure 23: CrossoverFree, Thermally Symmetric Flat Wind 


\section{0 / Critical Reviews Vol. CR77}

Experiments have been conducted at AMCOM to better define the effects of time-varying thermal gradients and related phenomena on the performance of IFOG sensing coils. ${ }^{47,48}$ A heat conduction model is used to analyze the nonreciprocity caused by time-varying thermal gradient in IFOGs. A comparison was made on the effects of radial thermal gradients on both quadrupolar and straight wound sensing coils in an IFOG breadboard for two types of polarization-maintaining fibers.

A literature review of the FOG technology advances in industry was conducted at AMCOM. The sensor coil was determined to be the primary cost driver in FOGs due to the extremely high cost ( $\$ 6-\$ 10$ per meter) of polarization-maintaining (PM) fibers. To best demonstrate the Army's requirement for a low-cost inertial navigation system, a depolarized FOG design that uses low-cost single mode (SM) fiber was developed. A 30-50-fold reduction in fiber cost of the sensing coil was realized in the depolarized design. An investigation was conducted to study the effects of a substantial size reduction in the fiber optic sensor coil and to develop and evaluate miniaturized sensor coil configurations that could perform over military environmental conditions. Issues associated with packaging IFOG components into a miniature volume were also addressed. The critical research and development activities conducted at AMCOM have resulted in a low cost, miniature fiber optic sensor coil design that is robust and thermally symmetric. A performance factor of better than two has been demonstrated for short $<$ 200 meters) low cost, SM fiber.

It was determined that the inherent fiber crossovers in the conventional precision wound coils tend to degrade the performance of the depolarized FOG. The number of crossovers in a precision wound coil is proportional to the product of the sensor coil height and the number of layers. The smaller the diameter, the more layers of optical fiber must be wound onto the coil form in order to maintain the fiber length while assuring high quality and performance stability of the FOG. Fiber crossovers, which are extremely large in number for ultraminiature coils, have a polarization scattering effect, which in turn creates a polarization nonreciprocal (PNR) error. These scattering sites provide for many (thousands) of birefringent delays, many of which will allow for recorrelation of PNR terms. Since there are thousands of crossovers in miniature coils, these crossovers have a large effect on bias performance.

A winding technique was devised to eliminate crossovers, and at the same time to be thermally symmetric (See Fig. 23). The significance of this development is two fold. First, the crossover-free method eliminates PNR bias errors for the depolarized FOG configuration which will lessen the need for more complex, expensive electronics. Secondly, the winding technique solves a 20 -year old problem that the entire FOG community has been investigating; namely, the advent of an advance fiber winding technique to eliminate the effect of time-varying thermal gradients in both the radial and axial directions of the fiber sensor coil. The elimination of bias errors created by thermal gradients will greatly reduce electronics, modeling, and cost. The conventional quadrupolar winding technique (Fig. 22), which is widely used by FOG developers, virtually eliminates the radial component of a time-varying thermal gradient but only slightly reduces the effect of the axial component. The thermally symmetric, crossoverfree winding technology eliminates the radial and axial components of a thermal gradient because of the completely symmetric design. The thermally symmetric, crossover-free technology will aid all FOG designers in optimizing fiber optic coils for applications requiring small or restricted volume at any performance regime. 
Novel Materials and Crystal Growth Techniques for Nonlinear Optical Devices / 21

The novel fiber optic sensor coil design and the efforts of the research team have revolutionized fiber optic sensor coil technology for Army applications as well as for a broad array of military and commercial applications. The demonstration of the patented (Patent \# 5,781,301) ultraminiature fiber optic sensor coil winding design has shown a substantial reduction in the noise and drift of depolarized FOGs. This technology breakthrough offers a very substantial benefit to the FOG industry.

\section{SUMMARY}

The results of the tremendous research efforts of the Government/industry team to develop fiber optics technology for military applications have been presented. Fiber

optic technology development programs for military applications have been very successful. More than 10 major Fiber Optic Guided Vehicle programs have been conducted in the Army, Navy and Air Force. Although there has not been a FOG-V fielded during the past 25 years, a host of advanced technology demonstrations have been achieved. Payout fibers with attenuation of less than $0.20 \mathrm{~dB} / \mathrm{km}$, light source level greater than $15 \mathrm{dBm}$, and a receiver sensitivity of less than $-40 \mathrm{dBm}$ have all been achieved under Government-sponsored programs. A fiber optic dispenser exceeding 50$\mathrm{km}$ fiber has been payed out from an aircraft. A $100-\mathrm{km}$ fiber optic data link has been demonstrated in the laboratory.

It was determined that the intrinsic pack stability could be improved through the use of fewer layers, stiffer bobbins and buffer coating materials, and modified winding tension profiles. Fewer layers of fiber were accomplished by the development of a thin-buffered $(200 \mu \mathrm{m})$ fiber.

Most of the dynamic fiber-pack instability problems were significantly reduced through advances in the fiber optic dispenser design. A proper winding tension profile resulted from the pack mechanics models and validation efforts. This was made possible by the non-destructive, length-dependent strain measurement unit. Optical signal loss induced by fiber bending at the peel point was all but eliminated by bend-insensitive fibers.

It was determined that an elevated temperature soak was required to relieve initial winding stress. Accelerated aging of fiber optic dispensers were conducted for the first time. The first military vehicle to demonstrate telecommunication fiber was an unmanned ground vehicle. First class facilities and instrumentation for fabricating and evaluating fiber optic dispensers were developed. The efforts to automate the fiber winding process resulted in a 10 -fold reduction in winding time. The successful demonstration of the non-conventional fiber optic gyroscope (FOG) sensor coil design that entirely eliminates the fiber crossovers, which are inherent in the conventional precision-wind method (traditionally used in the FOG community) was a key milestone in IFOG development.

\section{ACKNOWLEDGEMENTS}

The author would like to acknowledge the contributions to this review article from C. C. Sung, Anup Sharma, W. McCorkle, P. Jacobs, J. Meadows, R. Powell, J. E. Holzschuh, W. Culver, H. Wichansky, V. Kalomiris, C. Lofts, S. Burgett, J. Baeder, M. Steelman, J. 
Holt, T. Hester, J. Locker, P. Jezek, and B. Allan. The leadership and pioneering efforts of D. S. Fox in fiber-optic dispenser technology development are acknowledged.

\section{REFERENCES}

1. F. P. Kapron, D. B. Keck, and R. D. Maurer, Appl. Phys. Let., 17, 423 (1970).

2. W. Culver, et. al., "History of Optical Fiber Data Links for Tethered Weapons", Proceedings of DOD Fiber Optic Conference '90, McLean, VA, p. 234-238, 1992.

3. P. L. Jacobs, "Fiber Optic Data Link Development for the Army's Fiber Optic Guided Missile (FOG-M)", DOD Fiber Optic Conference, McLean, VA, p. 182-183, 1988.

4. W. C. McCorkle, R. E. Alongi, and G. P. Van Kirk, "Teleoperated Missiles as a Reconnaissance Asset on the Digital Battlefield,"

5. J. E. Holzschuh, "The Sea Ray Program," Proceedings of DOD Fiber Optic Conference ' 88 , McLean, VA, p. 184, 1988.

6. J. E. Holzschuh, "The Sea Ray Program," Proceedings of DOD Fiber Optic Conference '90, McLean, VA, p. 281-282, 1988.

7. A. R. Habayeb, "Navy Fiber Optic Data Link," Proceedings of DOD Fiber Optic Conference '90, McLean, VA, p. 272-280, 1990.

8. J. E. Holzschuh, "Fiber-Optic Data Links for Weapon Systems," NOSC Technical Document 2048, March 1991.

9. P. Jezek, "The Development of Fiber Spools for Underwater Payout," Proceedings of DOD Fiber Optic Conference '90, McLean, VA, p. 283-284, 1990.

10. D. S. Fox, "Ruggedized Cable Winding Trade Off Studies", Final Technical Report, Hughes Aircraft Company, Contract \# DAAK80-80-C-0595, Mar 1986.

11. A. Urano, M. Ishiguro, M. Shigematsu, et al., "Pure Silicon Core Dispersion-Shifted Single-Mode Fibers," $13^{\text {th }}$ ECOC, p. 175-178, Sep 1987.

12. M. W. Shute and H. T. Shang, "Optical Fiber for Tethered Vehicle Application," DOD Fiber Optics Conference, p. 187-191, Mar 1990.

13. T. C. Starkey and J. W. Suggs, "Reduced Mode-Field Diamater Single Mode Fiber for Specialty Applications," Proc. IW\&CC, p. 15-17, Nov 1988.

14. N. Kamikawa, "A Dual-Mode Optical Fiber for Long-Haul Transmission," Proceedings of DOD Fiber Optic Conference '90, McLean, VA, p. 253-254, 1992.

15. P. B. Ruffin C. C. Sung, "Excess Signal Loss in Precision-Wound Optical Fiber", SPIE Proceedings, Vol. 776, Optics and Optical Systems, Optics, Electro-Optics, and Sensors, 1987.

16. D. S. Fox, et al., Enhanced Fiber Optic Communication Link (EFOCL), Vol. 3, Environmental/ Mechanical Study, CECOM-TR-K543-F1 (1988). Report prepared by Hughes Aircraft Company.

17. D. Marcuse, Bell Syst. Tech. J., $\underline{51}, 329$ (1972).

18. P. B. Ruffin and C. C. Sung, "Temperature Dependence of Signal Loss in PrecisionWound Optical Fiber", SPIE Proceedings, Vol. 842, Fiber Optics Reliability: Benign and Adverse Environments, 1987.

19. J. P. Garmon, Automated Optical Fiber Winding System: Adhesive Investigation, Technical Report RG-CR-85-4, Contract No. DAAH01-83-D-A013, Jul 1984.

20. B. Allan, "Adhesive Development for Fiber Optic Payout Dispensers," Proceedings of DOD Fiber Optic Conference '90, McLean, VA, p. 245-247, 1992.

21. J. C. Holt, The Identification, Selection, Evolution, and Subsequent Development of the XP-156 Solventless Fiber Optic Dispenser Adhesive for the EFOGM System, Technical Report RD-MG-97-11, Mar 1997. 
Novel Materials and Crystal Growth Techniques for Nonlinear Optical Devices / 23

22. E. O. Rausch and P. B. Ruffin, "Fiber Optic Strain Sensing with RF Interferometric Techniques," SPIE Proceedings, Vol. 1170 Fiber Optic Smart Structures and Skins II, 1989.

23. S. J. Burgett, R. L. Light, P. B. Ruffin, and G. J. Counts, "Distributed Strain Measurements in Precision-Wound Optical Fibers Using Brillouin Optical Time Domain Reflectometry," SPIE Proceedings, Vol. 3330, p. 164-173, 1998.

24. S. J. Burgett, P. B. Ruffin, and R. L. Light, "Sensory and Calibration Issues Associated with Brillouin Optical Time Domain Reflectometry Measurements in Wound Optical Fibers,"

25. J. C. Holt, "Investigation of layer Tensions in the EFOGM Fiber Optic Dispenser Unit Using a Brillouin Optical Time-Domain Reflectometer," Technical Report RDMG-99-13, January 1999.

26. D. G. Padfield, "The Motion and tension of an Unwinding Thread, I" Proceedings of Royal Society of London, Vol. A245, p. 382-408, 1958

27. A. R. Barbin, "Dynamic Analysis of Fiber Optic Payout," System Dynamics Incorporated, Huntsville, AL, Report H-88-4, 1988.

28. S. Jones, P. B. Ruffin, J. Meadows, R. Berry, R. Eisentraut, and D. S. Fox, "Peel Point Loss During High Speed Payout of Optical Fibers," Proceedings of DOD Fiber Optic Conference '90, McLean, VA, p. 239-244, 1992.

29. T. Hester, A Force-And-Torque-Sensing Spool Fixture, Technical report RD-GC-8710, Jul 1987.

30. E. O. Rausch, et al., Technical Report RG-CR-85-3, "Automated Optical Fiber Winding System: System Design," 1984.

31. J. C. Hung, et al., Technical Report RG-85-23, "Development of Control Subsystems for an Automatic Fiber Winder, 1985.

32. M. L. Steelman, "Advances in Fiber Winding for FOG-V Applications," Proceedings of DOD Fiber Optic Conference '90, McLean, VA, p. 230-231, 1990.

33. C. D. Mabee, H. V. White, P. B. Ruffin, and J. C. Hung, U. S. Statutory Invention Registration, H502, "High Speed Precision Optical Fiber Winding System," 2 August 1988

34. M. L. Steelman, Chambered Spray Application System, Patent \# 5328512, 12 Jul 1994.

35. P. B. Ruffin, "Standards Issues in Accelerated Environmental Aging of Fiber Optic Payout Dispensers," Proceedings of DOD Fiber Optic Conference '90, McLean, VA, p. 207-208, 1990.

36. M. L. Steelman, "Environmental Methods to Determine Fiber Optic Aging Characteristics," Proceedings of DOD Fiber Optic Conference '90, McLean, VA, p. 209-210, 1990.

37. W. K. Burns, Optical Fiber Rotation Sensing, Academic Press, Inc., 1994

38. G. A. Alphonse, J. C. Connolly, N. A. Dinkel, S. L. Palfrey, and D. B. Gilbert, "Low Spectral Modulation High-Power Output from a New AlGaAs Superluminescent Diode/Optical Amplifier Structure", Appl. Phys. Lett., Vol. 55, No. 22, p. 2289, November 1989.

39. P. B. Ruffin and R. H. Smith, "Fiber Winding Approaches for EnvironmentallyRobust IFOG Sensor Coils," SPIE Proc., Vol. 1792 Components for Fiber Optic Applications, p. 179, September 1992.

40. Integrated Optical Circuit Consultant's SBIR Phase II Final Report, "Rugged/Low Cost Pigtailing Approaches for $\mathrm{LiNbO}_{3}$ Fiberoptic Gyroscope Chips II," September 1995. 
24 / Critical Reviews Vol. CR77

41. G. Chen, "Manufacturing Technology for Tactical Grade IFOGs," Proc. of the Workshop on Fiber Optics for Missile Applications, May 1996.

42. D. M. Shupe, "Thermally Induced Nonreciprocity in the Fiber Optic Interferometer," Appl. Opt. 19(5), 654 (1980).

43. N. J. Frigo, "Compensation of Linear Sources of Non-reciprocity in Sagnac Interferometers," Fiber Optic and Laser Sensors I, Proc. SPIE 412, p. 268 (1983).

44. B. Bednarz, J. A. Dankowych, G. Joslin, K. Jew, D. H. Lewis, and W. A. Young, "Optomechanical Systems for a Closed Loop Fiber Optic Gyroscope," Fiber Optic Gyros: $10^{\text {th }}$ Anniversary Conference, Proc. SPIE 719, p. 57 (1986).

45. R. Y. Liu, T. F. El Wailly, and R. C. Dankwort, "Test Results of Honeywell's FirstGeneration, High-Performance Interferometric Fiber-Optic Gyroscope," Fiber Optic Gyros: 15 th Anniversary Conference, Proc. SPIE 1585, p. 262 (1991).

46. P. B. Ruffin, C. Lofts, C. C. Sung, and J. Page, "Reduction of Nonreciprocity Noise in Wound Fiber Optic Interferometers," Opt. Eng. 33(8), 2675-2679 (1994).

47. C. M. Lofts, P. B. Ruffin, M. Parker, and C. C. Sung, "Investigation of the Effects of Temporal Thermal Gradients in Fiber Optic Gyroscope Sensing Coils," Opt. Eng. 34(10), 2856-2863 (1995).

48. J. Sawyer, P. B. Ruffin, and C. C. Sung, "Investigation of the Effects of Temporal Thermal Gradients in Fiber Optic Gyroscope Sensing Coils, part 2," Opt. Eng. 36(1), 29-34 (1997). 